\title{
Whole-Brain Vasculature Reconstruction
}

\section{Jerzy Krupinski*}

Department of Clinical Neurology, John Radcliffe Hospital, UK

The distinct association of the cerebrum's vascular system guarantees that it is sufficiently provided with oxygen and supplements. Be that as it may, in spite of this major part, an itemized reproduction of the brain wide vasculature at the slender level stays slippery, because of inadequate picture quality utilizing the best accessible procedures. Neuronal action depends on a complex system of veins that conveys oxygen and supplements for neuronal digestion. In spite of the basic significance of this framework, we don't have a finish topological comprehension of the slim system through which the trading of substances and metabolites happens. Mapping the fine anatomical subtle elements of vessels over the whole mouse cerebrum has been testing since it requires micrometer determination combined with quick procurement speeds to cover the whole specimen volume in a sensible day and age. Albeit substantial vessels in the entire mouse mind can be recognized non-obtrusively with an assortment of techniques, these strategies give a somewhat coarse determination. Other insignificant obtrusive techniques permit just poor representation of littler shallow vessels, without achieving slim level determination and without including profound regions. The fine system can be imagined at high resolutions by means of optical microscopy; be that as it may, profundity is restricted when gaining pictures from dark specimens. The improvement of optical clearing methods has permitted mouse organs, including the cerebrum, to be rendered totally straightforward, along these lines conquering this impediment. Among the optical methodologies accessible in blend with tissue clearing, light-sheet fluorescence microscopy (LSFM) offers the most astounding securing speed and is consequently especially alluring for recreating extensive volumes of tissues that would some way or another take too long to picture utilizing point-examining procedures like confocal microscopy or two-photon fluorescence microscopy (TPFM). Undoubtedly, clearing procedures combined with LSFM have been widely utilized for imaging fluorescently-marked segments in entire mouse organs, counting the perception of cerebrum vasculature. Imaging of the vasculature coordinate with LSFM yields datasets of 2-3 TB for each cerebrum and computerized investigation is in this manner fundamental for removing significant morphological data from this information. In any case, as a result of to a great degree extensive sums of information to be prepared and the problematic picture quality, cerebrum wide vascular system remaking at the hair like level has not been accomplished yet. Actually, vascular system remaking has been proficient just inside a part of mouse cerebrum cortex utilizing TPFM. The extraordinary outcome of this last work has been made conceivable by the utilization of a novel vascular recoloring strategy by which the entire vein lumen is loaded with a fluorescent gel the appropriateness of such a recoloring strategy to entire cerebrum acquisitions with LSFM isn't direct in light of the fact that it should be perfect with an entire mind clearing strategy. Recently, a strategy consolidating this marking approach with 3DISCO entire mind clearing was proposed. In any case, the destructive impact of the mounting medium required the utilization of a particular target focal point with low amplification and coarse determination. With the suitable changes on the clearing method, such mix between vessel lumen recoloring and CLARITY tissue clearing can be improved for examination of different organs, extending further the exploration fields which could profit of such vascular examination system.
*Corresponding author: Jerzy Krupinski, Professor, Department of Clinical Neurology, John Radcliffe Hospital, UK, Tel: +44 300304 7777; E-mail: jerzykrupinski.uk@rediffmail.com

Received September 20, 2017; Accepted October 06, 2017; Published October 17,2017

Citation: Krupinski J (2017) Whole-Brain Vasculature Reconstruction. J Vasc Med Surg 5: 346. doi: 10.4172/2329-6925.1000346

Copyright: (C) 2017 Krupinski J. This is an open-access article distributed under the terms of the Creative Commons Attribution License, which permits unrestricted use, distribution, and reproduction in any medium, provided the original author and source are credited. 